\title{
Dynamical aspects of motor and perceptual processes in schizophrenic patients and healthy controls
}

\author{
Andreas Keil ${ }^{\mathrm{a}, *}$, Thomas Elbert ${ }^{\mathrm{a}}$, Brigitte Rockstroh ${ }^{\mathrm{a}}$, William J. Ray ${ }^{\mathrm{b}}$ \\ a Department of Psychology, University of Konstanz, Konstanz, Germany \\ ${ }^{\mathrm{b}}$ Department of Psychology, Pennsylvania State University, University Park, PA, USA
}

\begin{abstract}
The present study examined the temporal stationarity of the performance of 16 schizophrenic patients and 16 controls matched for age and sex in a bimanual coordination task and a perceptual task. In the motor task, rhythmic finger oscillations (alternating activity of homologue muscle groups) at increasing speed levels resulted in two measures, the preferred oscillation frequency and the critical frequency at which phase transitions (change towards simultaneous activity of homologue muscle groups) occurred. A measure of local dimensional complexity (pointwise D2 or PD2), which is a measure of non-linear dynamics, was determined for the acceleration profiles of the subjects' movements. Schizophrenics exhibited less stable movement dynamics than controls in horizontal finger cycling, indicated by a lower ratio critical/preferred frequency (critical ratio) and by higher means and standard deviations of the pointwise D2. In vertical cycling, the critical ratio did not differentiate between groups, while PD2 means and standard deviations did. Groups also differed specifically in perception of two ambiguous figures (Schroeder stairs and Rubin vase). Schizophrenics showed significantly higher reversal rates for the Rubin vase and a differential perceptive in comparison to controls in the perception of the Schroeder stairs. Measures of perceptual and motor stability were unrelated, which suggests that perceptual and motor processes are not influenced by a common underlying mechanism.
\end{abstract}

Keywords: Schizophrenia; Movement coordination; Bistable figures; Non-linear dynamics; Chaos

\section{Introduction}

A potentially important new approach to describing dynamical processes, particularly those with signals that initially appear irregular or even random, is that of non-linear dynamics, also called

\footnotetext{
* Corresponding author. Present address: University of Konstanz, Fachgruppe Psychologie, Box D25, D-78457 Konstanz, Germany. Tel: +49 7531 884003; Fax: +49 7531 88289; e-mail: Andreas.Keil@uni-konstanz.de
}

chaos analysis. Such analyses allow us to determine changes in the dynamics of the system over time or the set of rules that characterize the system's state at a given time. More complex systems would be characterized by higher dimensionality or a higher rate of phase state transitions. Such approaches have been shown to be useful in characterizing a variety of processes, including physiology (Elbert et al., 1994) and motor behaviors (Haken et al., 1985). In schizophrenics, such measures reveal, for example, a higher rate of phase 
transitions (i.e., qualitative changes of the system's dynamical state) as well as a higher dimensionality and its variability in schizophrenics (Elbert et al., 1992; Koukkou et al., 1993; Rockstroh et al., 1997), indicating a lower stability of brain dynamics. Further, Yokoyama et al. (1996), using eye movements during reading, reported that schizophrenics display a fractal property of the inverse power law, whereas normals show an exponential distribution of a non-fractal nature. In contrast, in a study with normals, Kelso (1981, 1984) found a stable dynamic across all subjects during a motor task. In this task, individuals performed a rhythmic out of phase movement with each index finger. As they increased the speed of finger movements, a sudden in-phase transition appeared. The ratio of a person's critical frequency (i.e., the point of the sudden phase shift) and their preferred frequency (selected by the subject him/herself ) was computed and found to be similar across subjects. This task has not been studied with schizophrenics, and it is a purpose of the present study to extend Kelso's work into this area.

Schizophrenic individuals have been shown to display motor and perceptual dysfunctions in a variety of studies. In terms of motor behaviors, schizophrenics, in comparison to controls, show more pronounced intra-individual and interindividual variability and slower response on many tasks, including reaction time (Rist and Cohen, 1991), finger tapping (King, 1975), neuropsychological test performance (Bartfai et al., 1985) and repetitive movements (Jahn et al., 1995). Likewise, schizophrenics are shown to display perceptual dysfunctions on a variety of levels, including temporal resolution of stimuli (Kietzman, 1991), visual after-effects (Calvert et al., 1991; Schwartz et al., 1994; Harris, 1994) and perceptual organization (Bodner and Barak, 1995), although these results are not unequivocal (e.g., Rief, 1991; see also Steffy and Oakman, 1997 for a theoretical review of attentional functioning in schizophrenics).

One method for studying perceptual processing employs bistable figures, such as the Necker cube, Schroeder stairs or the Rubin vase, which may be seen in more than one aspect. Historically, this shift of perspective or reversal in figure perception has been explained by Koehler (1940) as the consequence of neuronal fatigue or saturation, and by Rock (1975) as the consequence of active selection and hypothesis testing by the viewer. In schizophrenics, bistable figures have been used to study perceptual stability or rigidity by measuring the number of perceived reversals (e.g., Hunt and Guildford, 1933; Eysenck, 1952; Nemor, 1953; Philip, 1953; Granger, 1957; D’Agata and Gaffuri, 1968). Calvert et al. (1988) measured duration of a percept rather than reversal rate and suggested significantly lower stability of schizophrenics' perceptual processes. One goal of the present study is to replicate and extend these findings and to apply non-linear dynamical measures to further understand irregular processes in schizophrenia.

Given that schizophrenics in comparison to normals show irregularities in response to motor and perceptual tasks, and since non-linear analyses of physiological processes such as EEG show higher dimensionality measures, we would expect that the application of non-linear measures to schizophrenic motor and perceptual responses would provide further clues to understanding schizophrenic dysfunction. In this manner, the characterization of perceptual and motor dynamics in schizophrenics can provide a further clue to the understanding of irregularities in schizophrenic processes demonstrated in formal thought disorders, cognitive slippage, or other characteristics. That is to say, transitions between thought patterns commonly seen in schizophrenic patients may reflect similar patterns to that of the perceptual and motor system, which may in turn be driven by common CNS mechanisms. However, few studies have examined the similarities in perceptual and motor processing dynamics displayed in the same population of schizophrenics. To this end, the present study examined motor instability and irregularity in schizophrenics using (a) Kelso's finger movement task and (b) reversible figures. Non-linear measures were applied to describe and quantify irregularity. Overall, we expected less stability to be found in the patient group in comparison to normals, as indicated by a higher rate of phase transitions in perceptual and motor dynamics. 


\section{Methods}

\subsection{Subjects}

Sixteen schizophrenic patients (12 men, 4 women, mean age $30.8 \pm 11.7$ years) were recruited from two regional psychiatric State hospitals. In terms of recruitment, the study was described to the patients during a weekly ward meeting and volunteers were solicited by the first author. The diagnosis according to DSM-IV was given by the ward psychiatrist or psychologist in charge, based on clinical files and extended personal contact with the patient. Of the 16 patients, 15 were diagnosed as paranoid-hallucinatory subtype (295.3) and one as disorganized (295.1). Duration of illness varied between 7 months and 23 years (mean duration $6 \pm 7$ years). All patients were under neuroleptic medication with a mean of $175 \pm 114 \mathrm{mg} /$ day, range $30-360 \mathrm{mg} /$ day chlorpromazine equivalent. Normal controls were recruited by advertisements in the local newspaper. From this procedure 16 healthy subjects (12 men, 4 women, mean age $30.5 \pm 12.4$ years) were selected to match to the patient group on age and sex. All subjects received a small financial bonus for their participation.

\subsection{Apparatus and materials}

Finger movements were recorded by means of a Kölner Vitaport System (Vitaport Systeme $\mathrm{GmbH}$, Erftstadt, Germany). This is an ambulatory system with 16 bit resolution. An accelerometer was fixed at the first joint of each index finger using medical adhesive tape. Values of acceleration and deceleration were digitized at a rate of $44.44 \mathrm{~Hz}$. A moving-average filter with a cut-off at $6.250 \mathrm{~Hz}$ was employed to eliminate frequencies reflecting tremor.

\subsubsection{Perceptual stimuli}

The Schroeder stairs and the Rubin vase (see Fig. 1) were used for bistable figures. Schroeder stairs allow a judgement of whether the stairs are seen from above or below, while the Rubin vase represents a figure which shifts in terms of figureground perception. The images were presented with constant illumination via paper cards forming
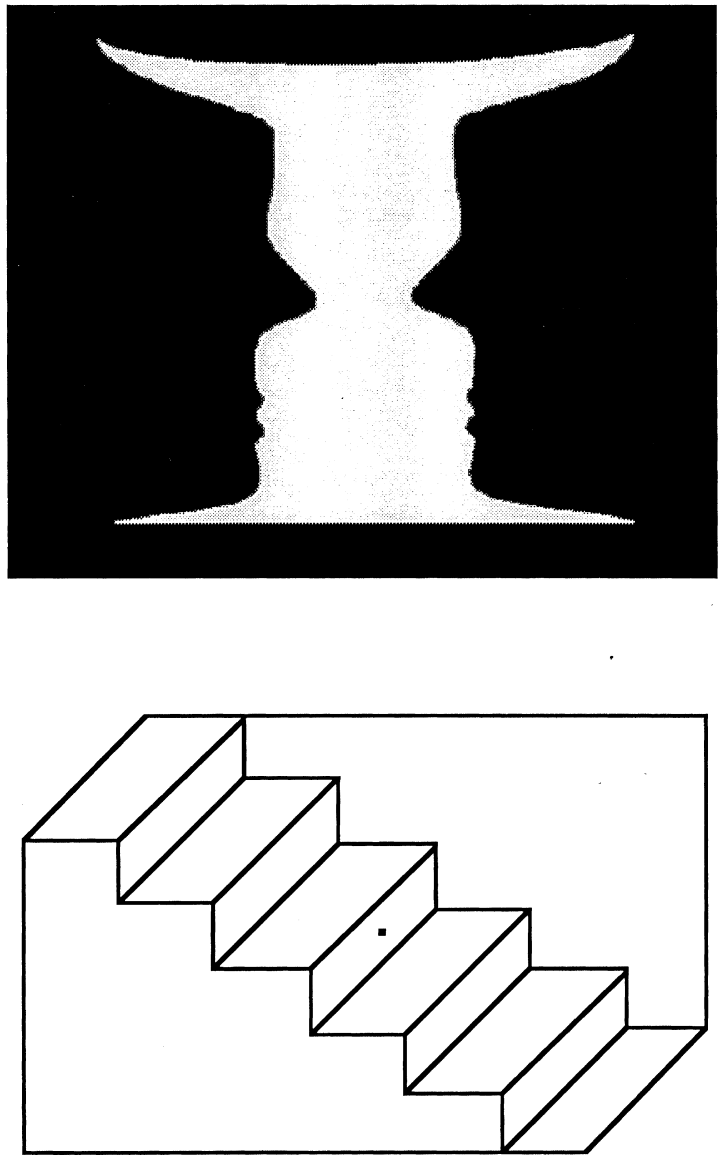

Fig. 1. The bistable stimuli: Rubin vase (top) and Schroeder stairs (bottom).

a visual angle of $10^{\circ}$. A $4 \mathrm{~mm}^{2}$ black fixation point appeared in the middle of each of the two stimuli. Subjects were told to fixate this point and to press a key whenever the perception of the figure changed. Key presses were sampled at $8 \mathrm{~Hz}$ and stored in a separate marker channel of the Kölner Vitaport System.

\subsection{Design and procedure}

Following Kelso's procedure (see Kelso, 1981), phase transitions were elicited in two types of movement patterns. (1) Horizontal movement: subject moves index fingers simultaneously left and right (alternating activity of homologue muscle 
groups). Increasing movement frequency leads to a switch into a symmetric pattern (synchronous activity of homologue muscle groups). (2) Vertical movement: subject moves index fingers up and down in opposite directions. When frequency is increased, there is a phase transition into a synchrony. Perceptual phase transitions were induced by presenting the bistable figures Schroeder stairs and Rubin vase.

The experimental session began with the perceptual task. After introducing the subject to the reversal phenomenon using non-ambiguous versions of the stimuli, a test trial was conducted in which reversals were observed and participants were trained how to signal perceived shifts. If the subject was not able to perceive a switch, the trial was terminated. Subjects were told that the perceptual shifts do not occur in everyone, and that a lack of perceptual shifts would not indicate any abnormality. In the bistable figure trials, subjects were first presented with both of the bistable figures for $90 \mathrm{~s}$ each.

Following the perceptual task, the motor task was explained to the subject. It was not demonstrated, to avoid imitation of cycling frequency. The subject was instructed to cycle 'at a rate that is most comfortable, not too slow and not too fast'. After $5 \mathrm{~s}$ the instruction 'a little faster' was given, and after another $5 \mathrm{~s}$ the experimenter instructed the subject to cycle 'even faster'. If no phase shift was observed up to this point in time, the instruction 'as fast as possible' was given. In order to test the reliability of the obtained data, a second identical trial was performed. Following the motor task, subjects performed the bistable figures again for $90 \mathrm{~s}$ in reverse order. Finally, subjects performed the second motor task under identical instructions as in the first task for two subsequent trials.

The sequence of tasks was counterbalanced across subjects to control for effects of fatigue, training, fluctuation of motivation or other nonspecific effects. In addition, marker responses indicating a subject's perception of one of the alternatives (e.g., press the key when you see the faces) were counterbalanced in order to eliminate effects of motor preference or fatigue.

\subsection{Data reduction and analysis}

A cross correlation between left- and right-hand accelerograms was computed to determine the time point at which in-phase finger cycling started. The presence of a shift into in-phase cycling was assumed whenever cross-correlation coefficients decreased from $r>|0.5|$ to $r<|0.15|$ (indicating desynchronization of finger movements) and subsequently returned to $r>|0.5|$ while cycling frequency was increased. In addition, observed phase transitions were recorded by the experimenter.

The frequency of finger cycling was determined for each subject by fast Fourier transformation (FFT) of the acceleration profiles using overlapping windows of 128 data points. The maxima of the resulting power spectral density (psd) function during the first 5-s interval (instruction 'most comfortable') were considered a measure of the preferred cycling frequency. The maximum of the psd function for the epoch immediately preceding the phase shift was taken as measure of the critical frequency. The individual ratio of critical and preferred frequency (critical ratio) was used as the measure of stability, with higher ratios indicating lower distractibility of coordination movements.

The pointwise correlation dimension (PD2) algorithm developed by Skinner (see Skinner et al., 1994) was used to determine the local dimensional complexity of the acceleration profiles (for a review of other applications see Elbert et al., 1994). Estimation of the PD2 includes scanning the time series with a time-lag $\tau$, which results in the reconstruction of the attractor according to the procedure proposed by Takens (1981). This attractor is assumed to represent the dynamics of the accelerogram in a multidimensional phase space. According to Skinner et al. (1994), the pointwise correlation dimension can be determined by computing the number of points $C$ within a sphere with the radius $r$ for each reference point $n$ ref, while $C(r, n, n \mathrm{ref}) \cong r^{\mathrm{PD} 2}$. Therefore, PD2 can be estimated as the slope of the $\log C(r, n, n$ ref $)$ in the $\log (r)$ domain, i.e., PD2 $\cong \log C(r, n, n$ ref $)$ $\log (r)$. Points that meet two criteria are considered as reference points ( $n$ ref). First, a linear segment in the $\log C(r, n, n$ ref $) / \log (r)$ plot within sufficient length is required, and second, the slopes have to 
converge with increasing attractor dimensionality. Given the properties described, the PD2 algorithm is able to detect changes in dimensional complexity indicating transitions between system states, while the standard deviation of a subject's PD2 values reflects stability of system states.

Differences between groups, trials and movement patterns were evaluated by means of an analysis of variance (ANOVA) with the betweensubjects factor GROUP (schizophrenic patients vs healthy controls) and the within-subjects factor TRIAL (one vs two) and cycling PATTERN (horizontal vs vertical). In order to obtain time series with sufficient length and a sufficient number of reference points, the PD2 was determined for time series consisting of two subsequent trials, under identical experimental conditions. Therefore the factor TRIAL was not used in the ANOVA performed on PD2 values. Differences between groups and trials in the perception of bistable figures were also evaluated by a 2 (group) by 2 (trial) ANOVA. A discriminant analysis based on the respective dependent measure was used for group classification. Linear univariate discriminant functions were computed using normal distribution method and Pearson product moment coefficients were employed to quantify the degree of stability of measures across trials and to determine the relation between clinical variables and dependent measures. All statistical analyses were performed using SAS for the PC, release 6.11 (SAS Systems Inc., 1995).

\section{Results}

All subjects displayed phase transitions in finger movements and reversals in the Rubin vase and the Schroeder stairs. Data from one subject, who reported dizziness, had to be rejected from the analyses of the Schroeder stairs perception.

Groups did not differ significantly with respect to the critical and preferred frequencies of finger movement $[F(1,15)=0.30]$. Vertical cycling was significantly faster in all subjects with respect to both critical frequencies $[F(1,15)=15.03$; $p<0.011]$ and preferred frequencies $[F(1,15)=$ 20.67; $p<0.01]$. A significant interaction GROUP $\times$ PATTERN in the critical frequencies
$[F(1,15)=7.46 ; p<0.05]$ indicates lower critical frequencies in schizophrenics when they executed the horizontal movement pattern.

The ratio of critical to preferred frequency suggested higher instability of the schizophrenics' movement process in the horizontal pattern [GROUP: $F(1,15)=9.38 ; p<0.01$; see Fig. 2 , left] but not in the vertical movement pattern $[F=0.22$; $\mathrm{df}=1,15 ; p<0.65$; see Fig. 2, right].

The PD2 means $[F(1,15)=10.42 ; p<0.01]$ and standard deviations $[F(1,15)=6.24 ; p<0.05]$ were higher for schizophrenics than for controls in both movement patterns. This main effect was not affected by the ordinal interaction of the factors GROUP $\times$ PATTERN $[F(1,15)=6.24 ; p<0.05]$. Therefore, the hypothesis of a more irregular and more distractable movement process in schizophrenics is supported by the analysis of local dimensional complexity.

Groups differed in the reversal rate for the Rubin vase $[F(1,15)=4.6 ; p<0.05]$ but not for the Schroeder stairs $[F(1,14)=0.41]$, i.e., patients exhibited a higher number of reversals than controls. A main effect of TRIAL indicated that reversal rate was enhanced in trial two for both groups $[F(1,15)=11.85 ; p<0.01]$. The time the Schroeder figure was seen from above was significantly shorter in schizophrenics than in controls $[F(1,14)=31.33 ; p<0.01 \text {, see Fig. } 3]^{1}$.

The individual PD2 mean values were significantly related to the medication status in patients, both for horizontal $(-0.54 ; p<0.05)$ and vertical $(-0.77 ; p<0.01)$ cycling, i.e., higher neuroleptic dosage covaried with lower PD2. Furthermore,

\footnotetext{
${ }^{1}$ In order to test the validity of these findings, analyses for perceptual and motor variables were performed on a subgroup of patients $(n=8)$ which was more homogeneous in terms of age (mean $=30$ years, $\mathrm{SD}=10$ years) and duration of illness ( mean $=5$ years, $\mathrm{SD}=1.5$ years, $\min =3$ years, $\max =7$ years), and their matches. The $F$ values and their probabilities for main effects of the ANOVA factor GROUP in the reduced sample for the following variables were as follows. Critical ratio for horizontal finger cycling: $F(1,7)=5.6, p<0.05$; critical ratio for vertical finger cycling: $F(1,7)=0.4$, n.s.; reversal rate of the Rubin vase: $F(1,7)=17.0, p<0.01$; reversal rate of the Schroeder stairs: $F(1,7)=0.36$, n.s.; time Schroeder stairs seen from above: $F(1,7)=16.6, p<0.01$. Thus, the group differences in this more homogeneous sample were as pronounced as reported for the complete sample.
} 

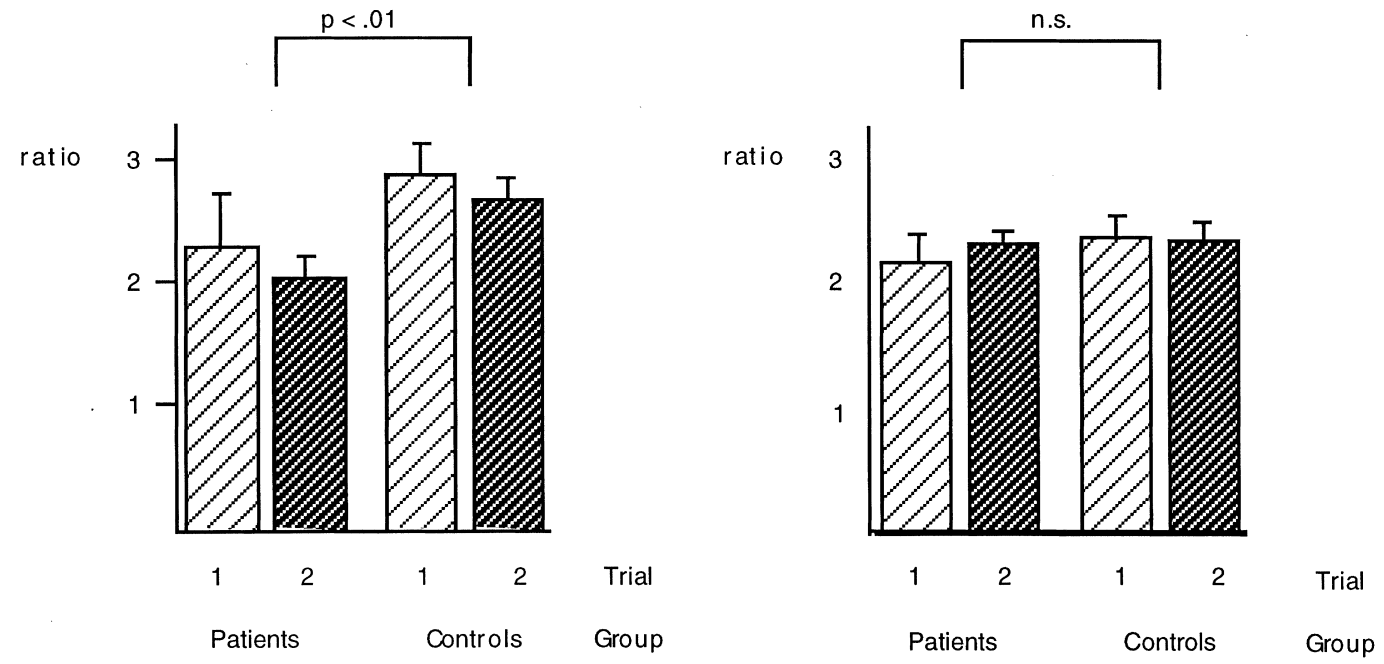

Fig. 2. Mean critical ratio (critical to preferred finger cycling frequency) for horizontal cycling pattern (left) and vertical cycling pattern (right). Bars indicate standard error $(N=32)$.

medication correlated with the time the Schroeder stairs were seen from above $(-0.56 ; p<0.05)$, i.e., higher neuroleptic dosage covaried with less time elapsed until change. Significant correlations between the duration of illness and the mean of individual PD2 in horizontal cycling $(-0.59$; $p<0.05)$ and the time the Schroeder stairs were seen from above $(-0.54 ; p<0.05)$ indicated that patients with a longer history of illness exhibited lower dimensional complexity of movement and

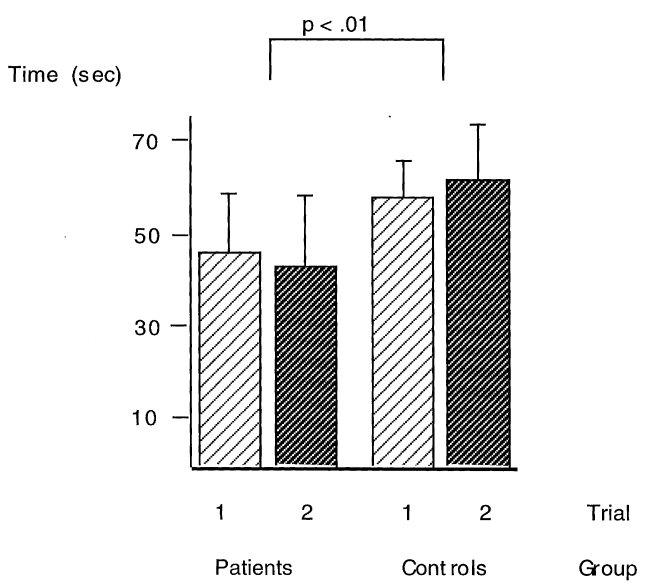

Fig. 3. Mean duration of Schroeder stairs seen from above for schizophrenic patients and normal controls $(N=30)$. Bars indicate standard deviation. less time perceiving the more realistic alternative of the stairs.

The measures of perceptual and movement stability were not significantly interrelated. However, in the patient group, the relation between the reversal rate of the Rubin vase and the ratio of critical and preferred frequency in horizontal cycling approached significance $(r=0.47 ; p<0.1)$. Thus, this relationship was in the opposite direction to that expected.

The highest percentages of correct classifications using linear discriminant functions for variables with significant group differences were found for the critical ratio in horizontal cycling $(78 \%$ correct classifications), the mean of the PD2 (81\%) and the time the Schroeder stairs were seen from above (80\%). The standard deviation of the PD2 (69\% correct classifications) and the reversal rate for the Rubin vase (64\%) were not as able to discriminate between groups. Discrimination of groups by critical ratio of horizontal finger cycling is illustrated by the bivariate scatter plot in Fig. 4 .

In the patient group, the mean of the stability coefficients (correlation between trials one and two) for all variables was 0.47 , as opposed to 0.75 in the controls. Likewise, intra-individual stability of data obtained by the patients was lower as compared to controls. Thus, as reported in previ- 


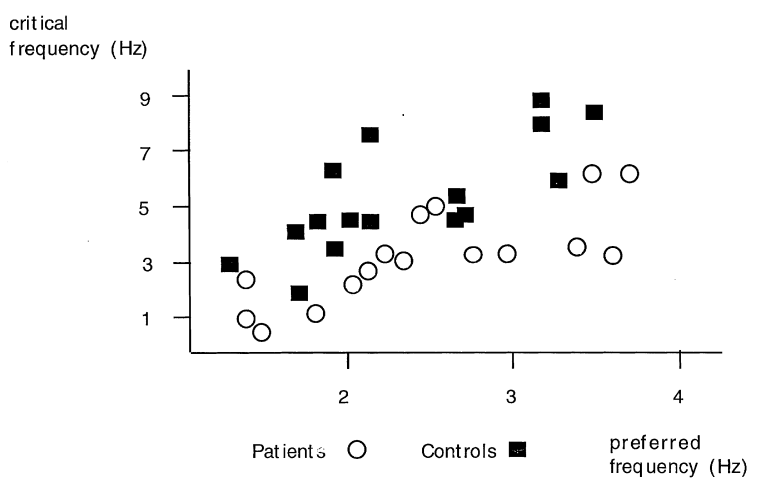

Fig. 4. Bivariate scatter plot of critical and preferred finger cycling frequencies for schizophrenic patients and normal controls $(N=32)$ in the horizontal cycling task.

ous studies (e.g., King, 1991; Rist and Cohen, 1991), motor responses were not as consistent in patients as in controls. Correlation coefficients indicating negative relations between performance in the two trials were found only in patients (critical ratio for horizontal cycling: $r=-0.17$; time stairs seen from above: $r=-0.41$ ). However, as there was no change in the group differences on each trial, findings seem not to be due to a lack of reliability in the experimental group.

\section{Discussion}

Given that schizophrenic individuals have been shown to display impaired motor and perceptual dysfunctions in a variety of studies (Elbert et al., 1992, 1994; Koukkou et al., 1993), we sought to characterize movement and perceptual dynamics in the schizophrenics using non-linear dynamical procedures. Since physiological studies using nonlinear procedures showed higher dimensionality measures in schizophrenics as compared to normals, we expected similar results with motor and perceptual processes. Using a simple finger motor task involving either horizontal or vertical movements, and a perceptual task involving reversible figures, we found differential results between the tasks suggesting that perceptual and motor processes in schizophrenia are not driven by a common CNS mechanism. In addition, important performance-related patterns were found to differ between schizophrenics and normals. Schizophrenic patients, as compared to controls, switched earlier from an out of phase into an in-phase pattern as they increased speed of finger movements in the horizontal but not the vertical direction. This resulted in a lower critical to preferred frequency ratio for horizontal movement. In terms of perceptual figure reversals, schizophrenics displayed higher reversal rates in the Rubin vase, but not in the Schroeder stairs.

The question arises as to the observed differences in horizontal and vertical movement patterns. One simple explanation is that vertical movements are more familiar and thus over-learned, since this type of movement is seen in various motor skills such as typing or playing a musical instrument, whereas horizontal finger movements are less well practiced. Further, given that there were no differences between the schizophrenic and control groups in preferred cycling speed, it would be difficult to suggest that the present findings resulted from less motivation on the part of the schizophrenic population. It is more likely that poor performance differences on the part of patients in the motor tasks reflect a specific kind of cerebral dysfunction still to be articulated.

In terms of motor processes, there are a number of proposals in the literature suggesting possible neural mechanisms underlying movement dysfunctions in schizophrenia, such as dysdiadochokinesis (Meehl, 1990), and impaired smooth pursuit eye dysfunctions (Levin, 1984a,b). Most mechanisms emphasize single component theories such as dysfunction of synaptic transmission or lack of frontal cortical control. King (1991) extended these views by suggesting that the slowed psychomotor speed found in schizophrenics results from impaired neural integration of various subcortical and cortical subsystems involved in motor behavior. It was our goal to extend this last aspect by applying concepts of non-linear dynamics to the problem. In the present study, not only critical ratios in horizontal cycling but also measures of dimensional complexity in both of the movement patterns allowed discrimination between schizophrenics and controls. Poor motor performance of schizophrenics can be expressed in terms of a lack of stability and a higher complexity in dynamic 
processes that coordinate actions. Zanone and Kelso (1992) suggested that these are determined mainly by intrinsic CNS tendencies and external requirements (e.g., cycling speed). This interpretation, as well as EEG/MEG results with schizophrenics, would lead one to consider a relationship between thought disorders and movement dysfunction (e.g., Manschreck et al., 1981; Bartfai et al., 1985) which we would expect to express in our perceptual processing results. In terms of perceptual figure reversals, schizophrenics in our study displayed higher reversal rates in the Rubin vase, but not in the Schroeder stairs. Therefore the present findings are not supportive of a theoretical connection between thought disorders and movement dysfunction.

A complete picture should, of course, describe the manner in which performance in using perceptual figures reflects different neural mechanisms and cognitive processes. This should be expected, since ambiguous figures such as the Rubin vase require processing of figure-ground discriminations, whereas geometric or reversible figures such as the Necker cube or Schroeder stairs require changes of perspective. Furthermore, reversal rate within a given time interval is not necessarily an optimal measure of visual stability. Other variables influencing the perception of bistable stimuli have been proposed, including the duration of the first percept prior to fluctuation or the latency in organizing the first percept (Lindauer and Baust, 1974). Replicating the work of Calvert et al. (1988), we showed in the present study that while reversal rate in the Schroeder stairs did not differentiate between groups, duration of perspective did, with the schizophrenics viewing the stairs longer from the less realistic perspective. This finding is further complicated by the finding that viewing perspective was related to dosage of medication, which would also be related to severity and/or duration of the disorder. Thus, further research would not only want to take these factors into consideration, but also include a variety of perceptual stimuli which would lead to more consistent findings in group differences between schizophrenics and control samples.

One concern of any study that uses medicated patients is the possibility that the results are moder- ated or mediated by the medication. We assumed that the variety of medications that our patients were on increases variability and reduced the possibility that one particular neuroleptic phenomenon could explain our results. Three additional points should be considered in the present study. First, we examined the scatter plots of the data by noting medication type and frequency. No consistent patterns were observed using this procedure. Second, it would be difficult to argue that our data could be explained by medication effects, since our data showed differential responding within-subjects. That is to say, the medication in the schizophrenics would have to have influenced horizontal movement differently from vertical movement. And third, although in the present study the amount of medication did influence performance on one visual illusion, the Schroeder stairs, the effect was to make the schizophrenics look more like, not less like, the controls. In this case, medication effects would have reduced, not increased, the differences we reported.

Theoretically, in order to describe processes underlying the perception of bistable figures, it is necessary to have a theory to account for, or model, the reversal phenomenon. Recent perceptual research favors a combination of fatigue and information-processing approaches (Phillipson and Harris, 1984). This can be further extended with the inclusion of non-linear dynamical models (e.g., Ditzinger and Haken, 1989). Within a so-called synergetic framework they were able to model some characteristics empirically found in the perception of bistable figures, for instance the increase of reversal frequency in the course of time. Such a model based on differential equations also was able to predict the relationship between group means of first perceived alternative percept and the individual mean duration of a given percept which is consistent with the empirical literature (Leeper, 1935; Lindauer and Baust, 1974). Furthermore, chaos or non-linear approaches have been shown to provide a useful framework for the study of other aspects of schizophrenia as well. Schizophrenic behavior which is often perceived as random may indeed be deterministic in the sense of being governed by a chaotic system including internal and environmental variables. As 
Schmid (1991) points out, there is more than one possible level of describing schizophrenia in terms of chaos. For example, Ciompi (1989), being concerned with questions of aetiopathogenesis and prognosis, used a model based on non-linear dynamics which accounts for the development of schizophrenic symptoms over the course of time. There are other studies using non-linear measures to explore differences between schizophrenics and normals regarding physiological time series, such as sleep EEG (Roeschke and Aldenhoff, 1993) or spontaneous EEG (Elbert et al., 1992). Our present results indicate that there might be further variables showing different dynamics in schizophrenics and normals which, in turn, confirm the efforts towards a non-linear approach in the study of schizophrenia. One main characteristic of such an approach would be the avoidance of assumptions about a single dysfunction by putting forward a model of abnormal interaction of involved brain subsystems and environmental components.

\section{Acknowledgment}

The authors would like to thank Dipl. Psych. Frank Fitz, Zentrum fuer Psychiatrie 'Die Weissenau' and Dr Hans Watzl, Zentrum fuer Psychiatrie Reichenau, for assistance in subject recruitment.

\section{References}

Bartfai, A., Levander, S.E., Nybaeck, H., Berggren, B.M., Schalling, D., 1985. Smooth pursuit eye tracking, neuropsychological test performance, and computed tomography in schizophrenia. Psychiatry Res. 15, 49-62.

Bodner, E., Barak, Y., 1995. Visual perception in schizophrenic patients. Eur. Psychiatry 10, 320-326.

Calvert, J.E., Harris, J.P., Phillipson, O.T., Babiker, I.E., Ford, M.F., Antebi, D.L., 1988. The perception of visual ambiguous figures in schizophrenia and Parkinson's disease. Intern. Clin. Psychopharmacol. 3, 131-150.

Calvert, J.E., Harris, J.P., Phillipson, O.T., 1991. Tilt aftereffect reveals early visual processing deficits in Parkinson's disease and in chronic schizophrenic patients on depot neuroleptic. Psychopathology 24, 375-380.

Ciompi, L., 1989. The dynamics of complex biological-psychosocial systems: four fundamental psycho-biological mediators in the long-term evolution of schizophrenia. Br. J. Psychiatry $155,15-21$.

D’Agata, G., Gaffuri, G., 1968. La percezione del cambiamento di prospettiva di una figura ambigua nella schizophrenia. Neuropsichiatrica 2, 357-363.

Ditzinger, T., Haken, H., 1989. Oscillations in the perception of ambiguous patterns. Biol. Cybern. 61, 279-287.

Elbert, T., Ray, W.J., Kowalik, Z.J., Graf, K.E., Birbaumer, N., 1994. Chaos and physiology. Physiol. Rev. 74, 1-47.

Elbert, T., Lutzenberger, W., Rockstroh, B., Berg, P., Cohen, R., 1992. Physical aspects of the EEG in schizophrenics. Biol. Psychiatry 32, 595-606.

Eysenck, H.J., 1952. Cyclothymia and schizothymia as a dimension of personality. Exp. J. Personality 20, 345-384.

Granger, G.W., 1957. Simple perceptual processes. In: Eysenck, H.J., Granger, G.W., Brengelmann, J.C. (Eds.), Perceptual Processes and Mental Illness. Oxford University Press, London.

Haken, H., Kelso, J.A.S., Bunz, H., 1985. A theoretical model of phase transitions in human hand movements. Biol. Cybern. $51,347-356$

Harris, J., 1994. The duration of the movement after-effect as an index of psychiatric illness. Perception 23, 1145-1153.

Hunt, J.M., Guildford, J.P., 1933. Fluctuation of an ambiguous figure in dementia praecox and in manic depressive patients. J. Abnorm. Soc. Psychol. 27, 443-452.

Jahn, T., Cohen, R., Mai, N., Ehrensperger, M., Marquardt, C., Nitsche, N., Schrader, S., 1995. Untersuchung der feinund grobmotorischen Dysdiadochokinese schizophrener Patienten: Methodenentwicklung und erste Ergebnisse einer computergesţtzten Mikroanalyse. Z. klin. Psychol. 24, 300-315.

Kelso, J.A.S., 1981. On the oscillatory basis of movement. Bull. Psychon. Soc. 18, 63

Kelso, J.A.S., 1984. Phase transitions and critical behavior in human bimanual coordination. Am. J. Physiol.: Reg. Integ. Comp. 15, R1000-R1004.

Kietzman, M.L., 1991. Information processing and schizophrenia. In: Nasrallah, H.A. (Ser. Ed.), Handbook of Schizophrenia, vol. 5: Steinhauer, S.R., Gruzelier, J.H., Zubin, J. (Eds.), Neuropsychology, Psychophysiology and Information Processing. Elsevier, Amsterdam, pp. 227-239.

King, H. E., 1975. Psychomotor correlates of behavior disorders. In: Kietzman, M.L., Sutton, S., Zubin, J., (Eds.), Experimental Approaches to Psychopathology, vol. IV: Methodological Issues. Academic Press, New York, pp. 421-450.

King, H.E., 1991. Psychomotor dysfunction in schizophrenia. In: Nasrallah, H.A. (Ser. Ed.), Handbook of Schizophrenia, vol. 5: Steinhauer, S.R., Gruzelier, J.H., Zubin, J. (Eds.), Neuropsychology, Psychophysiology and Information Processing. Elsevier, Amsterdam, pp. 273-301.

Koehler, W., 1940. Dynamics in Psychology. Liveright, New York.

Koukkou, M., Lehmann, D., Wackermann, J., Dvorak, I., Henggeler, B., 1993. Dimensional complexity of EEG brain 
mechanisms in untreated schizophrenia. Biol. Psychiatry 33, 397-407.

Leeper, R.W., 1935. A study of a neglected portion of the field of learning - the development of sensory organization. J. Gen. Psychol. 46, 41-75.

Levin, S., 1984a. Frontal lobe dysfunctions in schizophrenia: I. Eye movement impairments. J. Psychiatr. Res. 18, 27-55.

Levin, S., 1984b. Frontal lobe dysfunctions in schizophrenia: II. Impairments of psychological and brain functions. J. Psychiatr. Res. 18, 57-72.

Lindauer, M.S., Baust, R.F., 1974. Comparisons between 25 reversible and ambiguous figures on measures of latency, duration, and fluctuation. Behav. Res. Meth. Instr. 6, 1-9.

Manschreck, T.C., Maher, B.A., Rucklos, M.E., Vereen, D.R., Ader, D.N., 1981. Deficient motor synchrony in schizophrenia. J. Abnorm. Psychol. 90, 321-328.

Meehl, P.E., 1990. Toward an integrated theory of schizotaxia, schizotypy and schizophrenia. J. Pers. Disord. 4, 1-99.

Nemor, N., 1953. The alterations of an ambiguous figure in paretics and schizophrenics. J Abnorm. Soc. Psychol. 48, 445-447.

Philip, B.R., 1953. Reversals in the perception of lissajou figures by psychotics. Can. J. Psychol. 7, 115-126.

Phillipson, O.T., Harris, J.P., 1984. Effects of chlorpromazine and promazine on the perception of some multi-stable visual figures. Q. J. Exp. Psychol. 36A, 291-308.

Rief, W., 1991. Visual perceptual organization in schizophrenic patients. Br. J. Clin. Psychol. 30, 359-366.

Rist, F., Cohen, R., 1991. Sequential effects in the reaction times of schizophrenics: crossover and modality shift effects. In: Nasrallah, H.A. (Ser. Ed.), Handbook of Schizophrenia,
Vol. 5: Steinhauer, S.R., Gruzelier, J.H., Zubin, J. (Eds.), Neuropsychology, Psychophysiology and Information Processing. Elsevier, Amsterdam, pp. 241-271.

Rock, I., 1975. An Introduction to Perception. Collier McMillan, London

Rockstroh, B., Watzl, H., Kowalik, Z., Cohen, R., Sterr, A., Mller, M., Elbert, T., 1997. Dynamical aspects of the EEG in different psychopathological states in an interview situation. Schizophr. Res. 28, 77-85.

Roeschke, J., Aldenhoff, J.B., 1993. Estimation of sleep-EEG data in schizophrenics. Eur. Arch. Psychiatry Clin. Neurosci. 242, 191-196.

Schmid, G.B., 1991. Chaos theory and schizophrenia: elementary aspects. Psychopathology 24, 185-198.

Schwartz, B.D., Evans, W.J., Pena, J.M., Winstead, D.V., 1994. Visible persistence decay rates for schizophenics and substance abusers. Biol. Psychiatry 36, 662-669.

Skinner, J.E., Molnar, M., Tomberg, C., 1994. The point correlation dimension: performance with nonstationary surrogate data and noise. Integr. Physiol. Behav. Sci. 29, 217-234.

Steffy, R., Oakman, J., 1997. Attentional functioning in individuals diagnosed and at risk for schizophrenia. In: Burack, J., Enns, J. (Eds.), Attention, Development, and Psychopathology. Guilford Press, New York, pp. 318-349.

Takens, F., 1981. Detecting strange attractors in turbulence. Lecture Notes Math. 898, 366-381.

Yokoyama, H., Niwa, S., Itoh, K., Mazuka, R., 1996. Fractal property of eye movements in schizophrenia. Biol. Cybern. $75,137-140$.

Zanone, P.G., Kelso, J.A.S., 1992. Evolution of behavioral attractors with learning: nonequilibrium phase transitions. J. Exp. Psychol. Hum. Percept. Perform. 18, 403-421. 